\title{
ASMA INDUCIDA POR EJERCICIO Y ASPIRINA
}

\author{
Dra. Yolanda Noboa (t) y Dr. Cristóbal Sarzosa $(++)$ \\ (+) Servicio de Inmunología y Alergología. \\ $(++)$ Servicio de Neumalogía, Hospital die las FF. A.A., Quito
}

Asma por esfuerzo se entiende como la broncoconstricción producida por el ejercicio en un enfermo previamente diagnosticado de asma bronquial. Flayer en 1928 descubrió por primera vez las relaciones entre los distintos tipos de ejercicio y la mayor o menor capacidad de éstos para producir broncoconstricción. Desde 1960 se publican numerosos trabajos sobre asma inducida por ejercicio. Silverman y Anderson estudian 97 niños con asma bronquial a los que se les somete a un test de esfuerzo; se encuentra broncoconstricción en un $70 \%$ Fisiopatogénicamente se considera que durante el ejercicio en los enfermos asmáticos se produce una liberación de sustancias broncoconstrictoras o de enzimas precursoras. Su causa es discutida y hay la posibilidad de que la liberación de las sustancias broncoconstrictoras durante el ejercicio llegue al agotamiento de sus reservorios de manera que si un ejercicio que produce una broncoconstricción se repite a los treinta minutos, la respuesta constrictora es mucho menos importante. Mc Nally sostiene que la estimulación de los receptores orofaríngeos juega un papel importante puesto que anestesiando la orofaringe disminuye 0 desaparece la broncoconstricción. Shtruman encuentra que la respiración nasal disminuye la broncoconstricción luego del ejercicio y la respiración oral la aumenta. La temperatura dei aire inspirado y la humediad serían importantes en el desencadenamiento del asma por esfuerzo. Deal, señala que es el intercambio de calor del árboil traqueobronquial durante el ejercicio lo que determina la respuesta broncoconstrictora y que también la temperatura y la humedad del aire inspirado son fundamentales. Otros autores indican que pequeñas cantidades de histamina podrían ser liberadas luego del ejercicio, alcanzado una alta concentración local dentro de la mucosa respiratoria que podría causar broncoconstricción. El ejercicio generalmente causa un aumento de los basófilos circulantes por lo que es posible que pequeñas cantidades de histamina salgan hacia el plasma. Otros estudios demuestran niveles elevados del factor quimiotáctico de los neutrófilos inmediatamente después del ejercicio y mucho más tarde coincidentes con reacciones tardías inducidas por el ejercicio. El sistema nervioso autó 
nomo es considerado un factor impor tante en la patogénesis del asma. Se conoce que altas dosis de atropina inhiben el broncoespasmo inducido por el ejercicio en algunos asmáticos pero producen broncodilatación haciendo difícil la interpretación de los hallazgos. En estudios «invitro» ha sido demostrado que soluciones hiperosmolares causan liberación de meliadores químicos de los basófilos y de los mastocitos pulmonares; los bloqueadores de calcio inhiben el asma inducida por el ejercicio pero no afectan en el desencadenamiento del broncoespasmo provocado por inhalación de antígenos. Esto implica que las alteraciones en el transporte de calcio son importantes en la respuesta del sistema respiratorio a la sequedad y enfriamiento de las vías aéreas. Las pruebas funcionales con reto farmacológico de la ractividad de las vías aéreas se hacen con la inhalar ción de la histamina o metacolina.

El broncoespasmo desencadenado por la aspirina es debido probablemente a la capacidad de la aspirina de liberar específicamente histamina y otros mediadores farmacológicos de la hipereactividad inmediata. Los pacientes asmáticos tienen una probabilidad del 8 al $20 \%$ de experimentar un ataque asmático agudo después de la ingesta de aspirina; si tales pacientes tienen rinosinusitis o pólipos nasales la prevalencia aumenta del 30 al $40 \%$.

El broncoespasmo inducido por la aspirina puede ser el resultado de la generación de leucotrienos a través de la vía aiterna de la lipooxigenasa del ácido araquidónico, de la histamina y otros factores generados por la activación del complemento inhibiendo la vía de la ciclooxigenasa.

El ácido araquidónico es absorbido desde el intestino e incorporado a las membranas celulares como fosfolípidos por enzimas como la acíltransferasa; las prostaglandinas son sintetizadas luego de estimulación celular.

\section{MATERIALES Y METODOS:}

Se estudia cincuenta pacientes asmáticos de la consulta externa del Servicio de Inmunología Clínica y Alergia del Hospital General de las FF. AA. de Quito, con el propósito de investigar asma inducida por el ejercicio y la aspirina, en un período de ocho meses, desde enero a septiembre de 1985. La edad de los pacientes se cuenta entre el un año hasta los ochenta. Los parámetros de evaluación son: historia clínica, antecedentes atópicos tanto personales como familiares, la procedencia de los pacientes, presencia de eosinófilos en su secreción nasal, pruebas cutáneas de antígenos inhalables, valoración radiográfica de tórax y senos paranásales, pruebas funcionales respiratorias (PF R), valorando principalmente FEV1; FEV1/FVC y flujos de $25-75 \%$ y tomándose como pruebas positivas aquellas que presentaron alteraciones de broncoconstricción superiores al $15 \%$ de la prueba basal. Los pacientes fueron retados durante la realización de la PFR con $500 \mathrm{mg}$ de aspirina (ácido acetilsalicílico) y/o cinco minutos de ejercicio. Los datos obtenidos en el test con esfuerzo fueron valorados luego de cinco minutos de haberlo realizado. En lo 
referente a la aspirina la valoración de la severidad del broncoespasmo se realizó a la hora de ingesta de la misma.

A los cincuenta pacientes estudiados se los clasificó en cuatro grupos:

a) Pacientes que refirieron sintomatología relacionada con ejercicio.

b) Pacientes que refirieron sintomatología relacionada a la aspirina.

c) Grupo mixto con referencia sintomatológica relacionada con ejercicio y aspirina.

d) Pacientes cuya sintomatología clínica no fue relacionada con el ejercicio ni la aspirina.

\section{RESULTADOS:}

El $60 \%$ de los pacientes asmáticos estudiados relacionaron sus sintomas con el ejercicio, presentando de éstos solamente el $16 \%$ broncoespasmos superiores al $15 \%$ de la basal en las pruebas espirométricas. La broncoconstricción fue importante en las vías aéreas periféricas (VEF 25-75\%).

La relación existente con los demás parámetros de valoración se analiza en las tablas I, II, III y IV.

\section{TABLA I. RELACION ENTRE SINTOMATOLOGIA Y DATOS DE LABORATORIO EN LOS PACIENTES DEL GRUPO "A" (SINTOMATOLOGIA RELACIONADA CON EL EJERCICIO)}

\section{GRUPO a}

\begin{tabular}{|c|c|c|c|}
\hline Sierra & 17 & Costa & 13 \\
\hline $\mathrm{N}^{0}$ de pacientes & 30 & & $60 \%$ \\
\hline $\begin{array}{l}\text { Eosinofilia nasal } \\
\text { positiva } \\
\text { Test cutáneos }\end{array}$ & 11 Pctes. & & $36 \%$ \\
\hline positivos & 17 Pctes. & & $56 \%$ \\
\hline $\begin{array}{l}\text { Rx Sinusitis } \\
\text { Atopía familiar }\end{array}$ & 6 Pctes. & & $20 \%$ \\
\hline y/o personal & 26 Pctes. & & $86 \%$ \\
\hline
\end{tabular}

\section{PRUEBAS FUNCIONALES RESPIRATORIAS}

\begin{tabular}{lrl}
\hline PFR & Positivas a test de ejercicio & 5 Pctes. \\
\hline Disminución del VEF1 & $20 \%$ & 1 Pcte. \\
Disminución del FEV1/FVC & $0 \%$ & 0 Pctes. \\
Disminución del $25-75 \%$ & $80 \%$ & 4 Pctes. \\
\hline
\end{tabular}




\section{TABLA II. RELACION ENTRE SINTOMATOLOGIA Y DATOS DE LABORATORIO EN PACIENTES DEL GRUPO "B" (SINTOMATOLOGIA RELACIONADA CON LA ASPIRINA)}

\begin{tabular}{|c|c|c|}
\hline $\mathrm{N}^{\circ}$ de pacientes & 7 & $14 \%$ \\
\hline Sierra & Costa & 1 \\
\hline \multicolumn{3}{|l|}{ Eosinofilia nasal } \\
\hline Positiva & 1 Pcte. & $14 \%$ \\
\hline \multicolumn{3}{|l|}{ Test cutáneos } \\
\hline Positivos & 3 Pctes. & $42,8 \%$ \\
\hline $\mathrm{Rx}$ sinusitis & 0 Pctes. & $0 \%$ \\
\hline \multicolumn{3}{|l|}{ Atopía familiar } \\
\hline y/o personal & 5 Pctes. & $71 \%$ \\
\hline
\end{tabular}

PRUEBAS FUNCIONALES RESPIRATORIAS

\begin{tabular}{lrl}
\hline PFR & Positivas al reto de aspirina & 4 Pctes. \\
\hline Disminución del VEF1 & $75 \%$ & 3 Pctes. \\
Disminución del FEV1/FVC & $25 \%$ & 1 Pcte. \\
Disminución del $25-75 \%$ & $50 \%$ & 2 Pctes. \\
\hline
\end{tabular}

En este segundo grupo el $14 \%$ del total de pacientes presentan sintomatología relacionada con la ingesta ide aspirina, obteniendose broncoespasmo en el $57 \%$ en la disminución del VEF1 coma se demuestra en los cuadros anteriores descritos.

\section{DISCUSION Y COMENTARIOS:}

A los pacientes incluidos en el presente estudio se los dividió en cuatro grupos, de acuerdo a si su sintomatología se relacionaba con el ejercicio, con la aspirina, con los dos a la vez, y otro grupo el cual no tenía relación con el esfuerzo ni aspirina. En el primer grupo, el $16 \%$ de los pacientes presentaron broncoespasmo medible espirométrica- mente tras la estimulación con el ejercicio; en el segundo, el $57 \%$ presentó problemas de broncoconstricción con la ingesta de aspirina; los pacientes del cuarto grupo no fueron sometidos a reto con esfuerzo ni aspirina; además un $40 \%$ de ellos fueron niños menores de ocho años. Con estos datos se presume que hay mayor respuesta al reto con droga que con esfuerzo, probablemente debido a que la relación causa-efecto 
TABLA III. RELACION ENTRE SINTOMATOLOGIA Y EXAMENES DE LABORATORIO EN PACIENTES DEL GRUPO “C” (SINTOMATOLOGIA RELACIONADA CON EJERCICIO Y ASPIRINA)

\begin{tabular}{|c|c|c|c|}
\hline $\mathrm{N}^{9}$ de pacientes & 3 & & $6 \%$ \\
\hline Sierra & 3 & Costa & 0 \\
\hline Eosinofilia nasal & & & \\
\hline Positiva & 2 Pctes. & & $66.6 \%$ \\
\hline $\begin{array}{l}\text { Test cutáneos } \\
\text { positivos }\end{array}$ & 2 Pctes. & & $66.6 \%$ \\
\hline $\mathrm{Rx}$ sinusitis & 1 Pcte. & & $33.3 \%$ \\
\hline $\begin{array}{l}\text { Atopía familiar } \\
\text { y/o personal }\end{array}$ & 3 Pctes. & & $100 \%$ \\
\hline
\end{tabular}

PRUEBAS FUNCIONALES RESPIRATORIAS

PFR Positivas al reto con ejercicio y aspirina

Disminución del VEF1 $100 \%$

Disminución del FEV1/FVC

$0 \%$

Disminución del 25-75 \% $100-\%$

De los tres pacientes de este grupo el $33.3 \%$ presentó broncoespasmo inducido por esfuerzo y aspirina en las pruebas de reto medidas espirométricamente.

TABLA IV. RELACION ENTRE SINTOMATOLOGIA Y EXAMENES DE LABORATORIO EN PACIENTES DEL GRUPO "D" (NINGUNA RELACION CON EJERCICIO Y/O ASPIRINA)

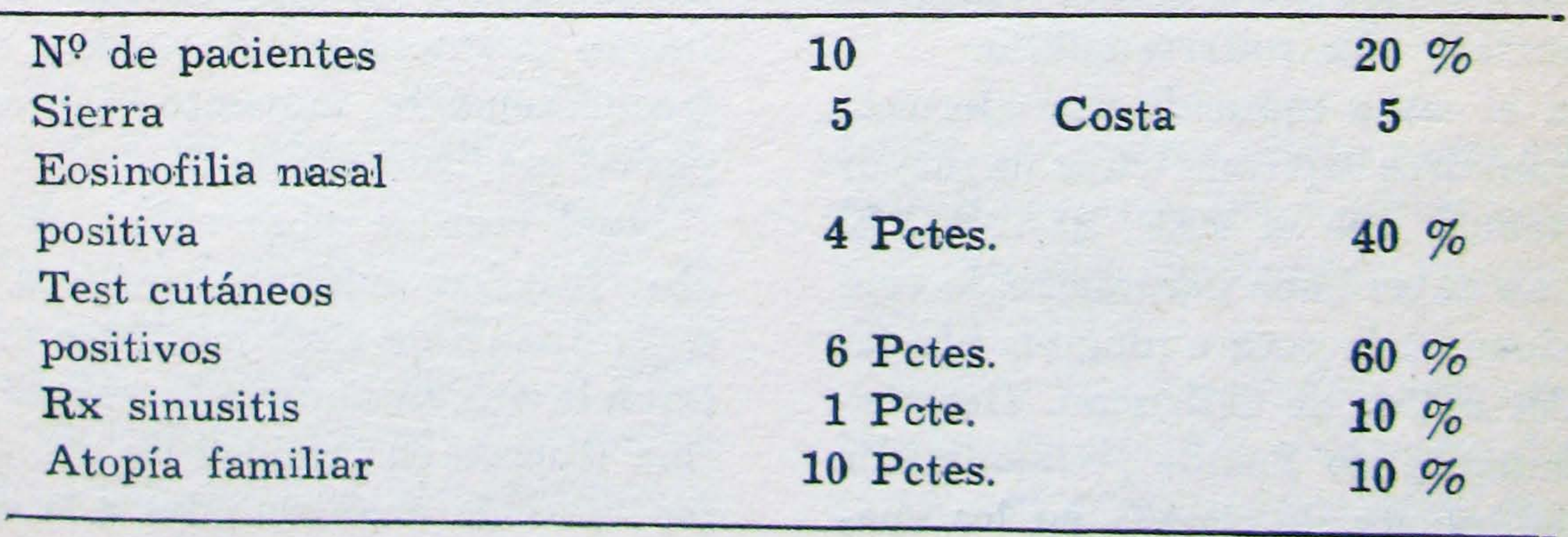


es más clara con los medicamentos. Además en el asma inducida por ejercicio hay otros factores como: tipo de ejercicio, tiempo de duración del mismo y condiciones en las que se realizan: niveles de ventilación, temperatura y humedad del ambiente. Algunos pacientes asmáticos durante el ejercicio incluso desarrollan broncodilatación transitoria mínima, posiblemente debido a la liberación de catecolaminas por incremento de actividad simpática. En los pacientes estudiados también se buscó respuesta broncoconstrictora a las dos y doce horas post-ejercicio sin obtener respuesta positiva. Posiblemente tomando un mayor número de casos, en el futuro se pueda obtener datos más concretos. En el caso de la aspirina se tienen cifras más altas con relación de causalidad, lo cual significa un mayor estado de alerta para el médico y el personal que realiza las pruebas de reto puesto que en el test de reto con aspirina a más de dificultad respiratoria con broncoespasmo se puede acompañar a otras manifestaciones clínicas que son consecuencia de los cambios producidos en los vasos y en la musculatura lisa bronquial. Estos pacientes suelen presentar urticaria, angioedema, hipotensión severa con pérdida del estado de conciencia y aún, muerte súbita.

Para el asma inducida por ejercicio los parámetros espirométricos de mayor importancia son el VEF 25-75\%. El VEF1 no es un buen parámetro, lo cual se traduce en la poca o ninguna afectación del índice de Tiffeneau. Desafortunadamente no hemos podido medir los «volúmenes de cierre» en los cua- les posiblemente tendríamos alteraciones más significativas. Por el contrario, en el asma inducida por aspirina, el mayor indicador es el VEF1, seguido muy de cerca por el VEF 25-75 y luego el índice de Tiffeneau. Para el grupo de personas que manifestaron relación sintomática con aspirina y esfuerzo, sólo una tercera parte de ellos presentaron alteraciones espirométricas luego del test de reto. Esto, como es lógico, se traduce en alteraciones del VEF1 y VEF 25-75. La mayor parte de los pacientes estudiados son procedentes de ia sierra, lo cual se explica por la localización de nuestro Centro Hospitalario.

La eosinofilia nasal positiva en los grupos a, b, y c se presentó en 14 pacientes. Porcentualmente más frecuente en el grupo mixto (c), luego en el de esfuerzo y apenas 1 de 7 pacientes hipersensibles a la aspirina mostró eosinofilia en secreción nasal.

En el grupo d (que puede considerarse como testigo) se presentó eosinofilia nasal en un $40 \%$. Tests cutáneos positivos se encontraron en el $56 \%$ de pacientes con relación a esfuerzo, lo cual podría significar que un porcentaje aproximadamente igual fueron en realidad asmas alérgicas y que en determinadas circunstancias ambientales el esfuerzo desencadenó el cuadro, o, más frecuentemente, aumentó el broncoespasmo mediado por Ig $\mathrm{E}$.

Igual cosa se observó en el grupo «b»: pruebas cutáneas positivas en el $42 \%$; cuatro de siete pacientes presentaron broncoespasmo inducido por aspirina (fueron en realidad los que tuvieron reacción anafilactoidea a la aspiri- 

TABLA V RELACION PORCENTUAL ENTRE ASMA ALERGICA
CON ESFUERZO Y/O ASPIRINA EN LOS 3 SUBGRUPOS INVESTIGADOS

GRUPO "a" Asma por esfuerzo

Asociación clínica

Verdadera asma por

ESFUERZO

Asma alérgica complicada

con esfuerzo

Asma alérgica $\mathrm{y} / \mathrm{o}$ infec-

ciosa con esfuerzo
30 Pctes.

$100 \%$

5 Pctes.

17 Pctes.

20 Pctes.
$16 \%$

$56 \%$

$20 \%$

GRUPO "b" Asma por aspirina

\begin{tabular}{lcc} 
Asociación clínica & 7 Pctes. & $100 \%$ \\
$\begin{array}{l}\text { Verdadera asma por } \\
\text { aspirina }\end{array}$ & 4 Pctes. & $57 \%$ \\
$\begin{array}{l}\text { Asma alérgica complicada } \\
\text { con aspirina }\end{array}$ & 3 Pctes. & $43 \%$ \\
\hline
\end{tabular}

GRUPO "c" Asma por esfuerzo y aspirina

\begin{tabular}{lcc}
$\begin{array}{l}\text { Asociación clínica } \\
\text { Verdaciera asma por aspirina }\end{array}$ & 3 Pctes. \\
$\begin{array}{l}\text { y esfuerzo } \\
\text { Asma alérgica complicada }\end{array}$ & 1 Pcte. & $100 \%$ \\
$\begin{array}{l}\text { por aspirina y esfuerzo } \\
\text { Asma alérgica y/o infecciosa }\end{array}$ & 1 Pcte. & $33 \%$ \\
por aspirina y E. & 1 Pcte. & $33 \%$ \\
\hline
\end{tabular}

na). En el grupo «c» también ocurrió algo parecido: uno de tres pacientes presentó estas modalidades de asma; el grupo de pacientes que presentó test cutáneo positivo correspondió al $60 \%$. Las radiografías de senos paranasales se realizó con el objeto de investigar la presencia de focos sépticos que estarian desencadenando los cuadros asmáticos $\mathrm{u}$ originando sindrome rino-sino-bronquial (SRSB).

El $20 \%$ de los pacientes con asma clínicamente asociada a esfuerzo, tuvo sinusitis, especialmente maxilar. No hubo 
pacientes con asma por aspirina con signos de sinusitis; tanto en el grupo mixto como en el grupo d, se encontró un paciente con sinusitis.

Nosotros hemos ampliado el término atopía no solamente a lo que clínicamente se entiende por ello; es decir antecedentes familiares $\mathrm{y} / 0$ personales de enfermedades alérgicas tipo I, sino a otros rangos de daño inmunológico global. Por esta circunstancia entre el 70 $-100 \%$ de nuestros pacientes manifestaron antecedientes positivos; dentro del término global se incluyó antecedentes de reacciones anafilactoideas a drogas, enfermedades autoinmunes, neoplasias hasta en tres generaciones. Posteriormente daremos a conocer datos más amplios a esta nueva concepción acerca de lo que significa atopía.

De lo anteriormente citado, se concluye que la relación entre esfuerzo, aspirina y asma alérgica o SRSB comprendida en estos parámetros, sería la que se sinteriza en la tabla V.

\section{RESUTMEN}

Se estudian cincuenta casos, tomados de la consulta externa del Servicio de Inmunología Clínica y Alergia del Hospital General de las FF. AA. de Quito. Con el objeto die investigar asma inducida por el ejercicio y la aspirina, en un periodo de ocho meses, en un grupo de pacientes cuya edad va diesde el un año a los ochenta años de edad, y con test a ejercicio y aspirina, midiendo FEV1; FEV1/FVC y FEV 25-75\%, y tomando como reto positivo a aquellos pacientes que hicieron broncocons- tricción superior al $15 \%$ de la basal espirométrica. Se dividieron a los pacientes de acuerdo a la relación sintomática con ejercicio y aspirina, un grupo mixto con sintomatología clínica producida por el ejercicio y la aspirina y además se tomaron en cuenta 10 pacientes que no refirieron relación con ejercicio ni aspirina. El $16 \%$ presentaron test positivos con alteraciones especialmente en las vías aéreas periféricas. De siete pacientes que manifestaron sintomatología clínica relacionada a la ingesta de aspirina el $75 \%$ presentaron test positivos. El 33\% de pacientes del grupo mixto presentan test positivos a la aspirina y esfuerzo.

Es importante el hecho de que no siempre se demuestra la etiología esfuerzo-aspirina del asma en los pacientes que así lo refieren, aunque es posible que factores repetidos de exposición ambiental variables como la humedad, temperatura etc., tengan una influencia directa en la aparición de la broncaconstricción en el asma inducida y/o complicada con el esfuerzo.

INCIDENCIA DE ASMIA EN EL HG-1

Asma de esfuerzo

$10 \%$

Asma por aspirina $8 \%$

Asma por ejercicio $\mathrm{y}$

aspirina

$2 \%$

Asma alérgica $52 \%$

Asma complicada con

infección o indeterminada 


\section{B I B L I O G R A F I A}

1 Bleecker, MD, Exercise-Induced Asthma, Clinics in Chest Medicine 5: 109-120, 1984.

2 Willis.: Asma Inducida por el ejercicio, Medicina Integral 5: 44 45. 1984.

3 Wiedeman, H. and Loke, J.: Exercise Testing in Occupational Lung Diseases, Clinics in Chest Medicine, 5: 157-172, 1984.

4 Loke J, Mahler D.: Exercise Impairment in Chronic Obstructive Pulmonary Disease, Clinios in Chest Medicine, 5 121-144 1984.
5 Gómez, C, Rubio M.: Asma de Esfuerzo Archivos de Bronconeumonía 16: 8-18 1982.

6 Durham R. and Kay, B.: The laboratory Cells in Asthma and Rhinitis, Clinics Inmunology and Allergy, 5: 1985.

7 Cummings, NMD, Morris, HMD, and Strunk RMD.: Failure of childnen with 'asthma to respond to daily aspirin therapy: Journal of Allergy and Clinical Inmunology, 71 245-249, 1983. 